\title{
Investigation of Cyg OB2 \#11(O5 Ifc) by Modelling its Atmosphere
}

\author{
Olga Maryeva ${ }^{1,3}$, Roman Zhuchkov² and Eugene Malogolovets ${ }^{1}$ \\ ${ }^{1}$ Special Astrophysical Observatory, Russian Academy of Sciences, Nizhnii Arkhyz 369167, Russia \\ ${ }^{2}$ Astronomy and Geodesy Department, Kazan (Volga region) Federal University, Kremlevskaya Street, 18, Kazan 420008, Russia \\ ${ }^{3}$ Email: olga.maryeva@ gmail.com
}

(Received August 24, 2013; ACCEPTEd March 05, 2014)

\begin{abstract}
We continue the study of O-supergiants belonging to the association Cyg OB2 using moderate-resolution spectra. In this paper we present results of the modelling of the stellar atmosphere of Cyg OB2 \#11. This object belongs to the spectral class Ofc, which was recently introduced and is yet small in numbers. Ofc class consists of stars with normal spectra with CIII $\lambda \lambda 4647,4650,4652$ emission lines of comparable intensity to those of the Of-defining lines NIII $\lambda \lambda 4634,4640,4642$. We combined new spectral data obtained by the 1.5-m Russian-Turkish telescope with spectra from MAST and CASU archives and determined physical parameters of the wind and chemical composition of the stellar atmosphere using CMFGEN code. The estimated nitrogen abundance is lower than one in atmospheres of 'normal' O-supergiants (i.e. O4-6 supergiants without additional spectral index ' $n$ ' or 'c') and carbon abundance is solar. Also we find an excess in silicon. We present an illustrative comparison of our modelling results with current Geneva evolutionary models for rotating massive stars. The position on the Hertzsprung-Russell diagram corresponds to the star mass of about $50 \mathrm{M}_{\odot}$ and age about $4.5 \mathrm{Myr}$. Moreover, we carried out the high angular resolution $(\sim 0.02 \operatorname{arcsec})$ observations on the Russian 6-m telescope aiming to find weaker companions of this star, which did not reveal any.
\end{abstract}

Keywords: stars: atmospheres - stars: early-type - stars: evolution - stars: fundamental parameters - stars: individual: Cyg OB2 \#11 - stars: winds, outflows

\section{INTRODUCTION}

Improvement in telescope detection limits, technical evolution of detectors, and emergence of huge databases of observational data resulted in more careful spectral classification of objects and provision of new spectral subclasses. Walborn et al. (2010) proposed to introduce a new subclass Ofc to denote O-stars with comparable intensity of CIII $\lambda \lambda 4647,4650,4652$ and NIII $\lambda \lambda 46634,4640,4642$ lines. This phenomenon is often observed at spectral type $\mathrm{O} 5$ at all luminosity classes, but preferentially in some associations or clusters and not the others (Walborn et al. 2010). For today, 18 Galactic O-stars are classified as Ofc.

Although Ofc class was introduced recently, $\mathrm{CNO}$ dichotomy is well known among O-stars (Walborn et al. 2004; Walborn 2003, 1976 and references therein). Anticorrelations of $\mathrm{N}$ versus $\mathrm{C}$ and $\mathrm{O}$ and correlations with $\mathrm{He} / \mathrm{H}$ have encouraged interpretations in terms of mixing of CNO-cycled material into the atmospheres and winds of massive stars. The mixing depends on the rotation rate and increases at low metallicity (Maeder \& Meynet 2010). But are Ofc stars related to CNO dichotomy only? With the advent of the Ofc class of stars the following questions arose:

- Whether it is related only to the CNO-cycle and mixing processes in the star itself or it arises under the influence of a general dynamical evolution of clusters or associations?

- How does multiplicity of objects influence the excess of carbon?

- Are there differences between the physical parameters of Ofc stars and Of stars?

- How should an Ofc star evolve further?

To obtain more data and to estimate more parameters of Ofc stars are important steps for better understanding of the nature of these objects. 
Cyg OB2 $\# 11^{1}$ is one of the Galactic supergiants located near the northern border of the Cyg OB2 (VI Cygni) association. This association was first noticed more than half a century ago by Münch and Morgan (1953). It still attracts the attention of researchers due to the large number of Ostars and extremely high, heterogeneous interstellar reddening. Star \#11 was immediately recognised as a member of the association (Münch and Morgan 1953). The first spectroscopy of the brightest stars belonging to Cyg OB2 (including \#11) was performed by Johnson \& Morgan (1954). They classified Cyg OB2 \#11 as O6f. Later Walborn (1973) classified the star as $\mathrm{O} \mathrm{If}_{+}$using spectrograms obtained at Kitt Peak National Observatory. Massey \& Thompson (1991) performed its CCD photometry in three $(U, B, V)$ bands. Cyg OB2 \#11 is included in their catalogue under number 734 (MT91 734). Stellar magnitude in $V$ band is $V=10.03 \mathrm{mag}$ (Massey \& Thompson 1991). As of now, Cyg OB2 \#11 is classified as O5.5Ifc (Walborn et al. 2010; Sota et al. 2011). Kobulnicky et al. (2012) found that Cyg OB2 \#11 is a singlelined spectroscopic binary (SB1 type).

The spectrum of Cyg OB2 \#11 was modelled previously by several groups. Initially, by means of numerical modelling Herrero et al. (1999) determined the parameters of Cyg OB2 \#11 (effective temperature, luminosity, $\log g$ and helium abundance). They compared the spectral line profiles of $\mathrm{H}, \mathrm{He} \mathrm{I}$, and $\mathrm{He}$ II with the line profiles synthesised for a large set of non-local thermodynamic equilibrium (NLTE) plane-parallel, hydrostatic model atmospheres. Herrero et al. (2001) measured terminal velocity of the wind employing resonance lines in the ultraviolet range. Then Cyg OB2 \#11 was modelled by Herrero et al. (2002) using the FASTWIND (Santolaya-Rey, Puls, \& Herrero 1997; Puls 2005) code. In that work the mass-loss rate and velocity law in the stellar wind have been determined. Finally, Mokiem et al. (2005) for the first time applied the automated fitting method and clarified the physical parameters of Cyg OB2 \#11. It is worth noting that the mass-loss rate was determined by Herrero et al. (2002) and Mokiem et al. (2005) without taking clumping into account. Inhomogeneities in the winds of the stars were studied in the article Puls et al. (2006). Based on a simultaneous modelling of $\mathrm{H} \alpha$, infrared, millimetre, and radio observations authors concluded that clumping is three to six times stronger in the lower wind, where $\mathrm{H} \alpha$ forms, compared with the outer wind, where the radio continuum originates.

In the next section, we describe the observational data and their processing. In Section 3 we will tell about the construction of the model, discuss the results, and compare them with previous works. Section 4 is devoted to determination of chemical composition of the atmosphere of Cyg OB2 \#11, while Section 5 shows locations of Cyg OB2 \#11 on the different diagrams. The search for companions of Cyg OB2 \#11 is described in Section 6. The conclusions are presented in Section 7.

\footnotetext{
${ }^{1} \alpha=20: 34: 08.52 \delta=+41: 36: 59.36$ according to SIMBAD http://simbad.ustrasbg.fr/simbad/.
}

\section{OBSERVATIONAL DATA}

We have combined spectra of Cyg OB2 \#11 from the archives of 4.2-m William Herschel Telescope $(W H T)^{2}$ with new data obtained on the 1.5-m Russian-Turkish telescope (RTT150). Archival spectra were obtained with the ISIS (the Intermediate dispersion Spectrograph and Imaging System) instrument of the WHT in July 1995 and in September 1998. R600B and R1200R gratings were used for observations. The spectral ranges are 4000-4800 $\AA \AA$ and 6350-6750 $\AA \AA$, and spectral resolutions are $\sim 3 \AA$ and $\sim 1.5 \AA$, correspondingly. Detailed description of these data can be found in paper by Walborn \& Howarth (2000). We processed the data using standard procedures for a long-slit spectroscopy.

Echelle spectrum of Cyg OB2 \#11 was obtained in November 2012 with the TFOSC (TUBITAK Faint Object Spectrograph and Camera) instrument, installed in the Cassegrain focus of RTT150. The spectral resolution is $\lambda / \Delta \lambda=2500$ and spectral range is $4200-8000 \AA \AA$. DECH software package was used for the data reduction and analysis (Galazutdinov 1996). DECH package includes all the standard stages of echelle data reduction process. Methods of observations and data reduction are the same as described by us in the paper (Maryeva \& Zhuchkov 2012). Besides, investigated object was observed in February 2013 on the same instrument with comparable integration time and image quality. Total exposure time was $80 \mathrm{~min}$ in both cases. No significant differences were found between the spectra obtained in 2012 and 2013 at an interval of $80 \mathrm{~d}$. In the overall spectrum the signal-to-noise ratio per sampling element is $S / N=100$ in the blue part (5000 $\AA$ ) and 200 in the red part (7000 $\AA$ ).

Moreover we have performed observations on the 6-m telescope of Special Astrophysical Observatory (SAO) with the speckle interferometer during December 2012 to look for close components at distances $0.02-4$ arcsec. These observations are described in more detail in Section 6.

We investigated the object in the UV range using the spectra obtained by the Hubble Space Telescope (HST) with the space telescope imaging spectrograph (STIS) and published by Herrero et al. (2001). These data were taken from the Multimission Archive at STScI (MAST) ${ }^{3}$. The spectral range of these data is $1150-1700 \AA \AA$, and the spectral resolution is $\lambda / \Delta \lambda \sim 1000-1500, S / N=19$.

\section{MODELLING}

In our work atmospheric parameters of Cyg OB2 \#11 are determined using both ultraviolet and optical data simultaneously. We have used the CMFGEN atmospheric modelling code (Hillier \& Miller 1998). This code solves radiative transfer equation for objects with spherically symmetric extended

\footnotetext{
${ }^{2} \mathrm{http}: / /$ casu.ast.cam.ac.uk/casuadc/ingarch/query. This paper makes use of data obtained from the Isaac Newton Group Archive which is maintained as part of the CASU Astronomical Data Centre at the Institute of Astronomy, Cambridge.

${ }^{3}$ http://archive.stsci.edu/.
} 
outflows using either the Sobolev approximation or the full comoving-frame solution of the radiative transfer equation. CMFGEN incorporates line blanketing, the effect of Auger ionisation, and clumping. Every model is defined by a hydrostatic stellar radius $R_{*}$, luminosity $L_{*}$, mass-loss rate $\dot{M}$, filling factor $f$, wind terminal velocity $v_{\infty}$, stellar mass $\mathbf{M}$, and by the abundances $Z_{i}$ of included elementary species. Although in FASTWIND ions of different chemical elements are taken into account, FASTWIND models computed by Herrero et al. (2002); Mokiem et al. (2005) assumed a solar mix of metals, and therefore could not be used to determine the chemical composition of stellar atmosphere. As a result, in our work we for the first time determine the chemical abundance of the atmosphere of Cyg OB2 \#11.

In the previous studies the absolute magnitude $\left(\mathrm{M}_{\mathrm{v}}\right)$ and bolometric corrections $\left(\mathrm{BC}_{\mathrm{v}}\right)$ were employed to estimate the luminosity. Numerous tabulations of $\mathrm{BC}_{\mathrm{v}}$ and $\mathrm{M}_{\mathrm{v}}$ for OI 5 existing in the literature sometimes differ significantly. For example, the absolute magnitude of Cyg OB2 \#11 in the work of Massey \& Thompson (1991) is $M_{v}=-6.9$ mag, in articles of Mokiem et al. (2005) and Herrero et al. (1999) it is $\mathrm{M}_{\mathrm{v}}=-6.51 \mathrm{mag}$, while in Puls et al. (2006) $\mathrm{M}_{\mathrm{v}}=-6.67 \mathrm{mag}$. At the same time, the absolute magnitude of O5 I class stars is $\mathrm{M}_{\mathrm{v}}=-6.33 \mathrm{mag}$, according to calculations of Martins, Schaerer, and Hillier (2005), the same value as assumed by Kiminki et al. (2007) for Cyg OB2 \#11. We will use other method of determining the luminosity. To accurately determine the luminosity of the object, the magnitudes of the star were calculated in the $U, B, V$, and $R$ filters from the model spectra and compared with observations. In order to obtain the magnitudes for the model spectra, we first recomputed the fluxes for the distance to the Cyg OB2 association (1.5 kpc according to Kiminki et al. (2007); Mel'nik \& Dambis (2009)). The resulting fluxes were corrected for the interstellar extinction. The value of the interstellar extinction $A_{v}=5.4$ also was taken from Kiminki et al. (2007). After this, the calculated spectra were convolved with the transmission curves of the standard $U, B, V$, and $R$ filters. Thus, we have calculated the grid of models with different luminosities and compared them with observations to derive the luminosity of the object.

A recent study by Kobulnicky et al. (2012) has shown that Cyg OB2 \#11 is a single-lined spectroscopic binary (SB1 type). Therefore, we decided to model Cyg OB2 \#11 as a single object. To determine the parameters of the Cyg OB2 \#11 atmosphere we chose the model most resembling the observational spectrum from the calculated grid of models of O-stars (Maryeva \& Zhuchkov 2012; Maryeva, Klochkova, \& Chentsov 2013a) and then started to refine the parameters of this model. We determined the effective temperature using the lines of $\mathrm{He}$ II $\lambda 4541.59,5411.52 \AA$ and He I $\lambda 4471.5,5875.66 \AA$, as well as the lines of nitrogen NIII $\lambda \lambda 4634.0,4640.6 \AA$ and weak absorptions of NIV $\lambda \lambda 5200.60,5204.28 \AA$. In the spectrum Cyg OB2 \#11 there are no lines of NV $\lambda \lambda 4604.16,4620.5 \AA$ that indicates that $T_{\text {eff }}$ is below $40000 \mathrm{~K}$.
The non-clumped mass-loss rate $\left(\dot{M}_{\text {uncl }}\right)$ is related to the clumped $\left(\dot{M}_{\mathrm{cl}}\right)$ by the relation $\dot{M}_{\text {uncl }}=\dot{M}_{\mathrm{cl}} / \sqrt{f_{\infty}}$, where $f_{\infty}$ is the volume filling factor at infinity. We investigated the effect of clumping on intensities of lines. We have stopped at a value $f_{\infty}=0.08$ which well describes resonance lines of silicon SiIV $\lambda \lambda 1393.75,1402.77 \AA$, and $H_{\alpha}$ line.

Figures 1 and 2 present the comparison of the observed spectrum of Cyg OB2 \#11 with the best model in the optical and the ultraviolet region. For comparison, Table 1 gives the parameters of Cyg OB2 \#11 derived by Herrero et al. (2002) and Mokiem et al. (2005). The temperature difference is within error limits. Obvious difference in the luminosity is due to the difference in estimations of distance to the star. In the previous works distance modulus is suggested to be equal to $11.2 \pm 0.1$, which corresponds to $\approx 1.7 \mathrm{kpc}$. In the present work, as stated above, we assume that the distance is $1.5 \mathrm{kpc}$ (Mel'nik \& Dambis 2009).

Most probably the differences in the mass-loss rate are due to taking clumping into account in our computations. Puls et al. (2006) also have measured the mass-loss rate taking clumping into account. They determined that massloss rate is $\dot{M}_{\mathrm{cl}}=(5 \pm 0.5) \cdot 10^{-6} \mathrm{M}_{\odot} \mathrm{yr}^{-1}$ and $f=1$ for the region where $H_{\alpha}$ is formed. Therefore $\dot{M}_{\mathrm{cl}}$ equal to $\dot{M}_{\text {uncl }}$ in this region. And our value of unclumped mass-loss rate is consistent with estimation Puls et al. (2006).

$\beta$ is the exponent in the velocity law describing the increase of velocity with radius. With the decrease of $\beta$ the depths of absorption lines vary and the widths of He II 4685.7 and $H_{\alpha}$ wings increase. In our calculations we stopped at $\beta=1.3 \pm 0.1$. This value is higher than the value of the $\beta$ parameter obtained for Cyg OB2 \#11 before (Table 1), but it is comparable to the $\beta$ parameters found for other O-class stars using CMFGEN (Table 3 ).

\section{CHEMICAL COMPOSITION}

Calculated abundances of the basic elements are given in Table 2. During the modelling we had difficulties with the description of He II 4685.7 line. This line is sensitive to changes in temperature and in wind conditions - clumping and mass-loss rate. At the outset of the modelling we supposed that $\mathrm{N}_{\mathrm{He}} / \mathrm{N}_{\mathrm{H}}=0.2$ (by number). We determined effective temperature through the $\mathrm{He}$ II to $\mathrm{He}$ I ratio and the mass-loss rate through NIII $\lambda \lambda 4634.0,4640.6 \AA$ and CIII $\lambda \lambda 4647,4650,4652 \AA$ lines, as well as $H_{\alpha}$ and $H_{\beta}$ lines in the way so $H_{\alpha}$ is in emission and $H_{\beta}$ in absorption, and as a result we got very strong He II 4685.7 line. To decrease the intensity of this line we have significantly reduced the mass fraction of helium in the model down to $16-28 \%$, which is $0.05-0.1$ fraction by number of atoms. Herrero et al. (2002) determined the abundance of helium as 0.09 (the He abundance by number of particles relative to $\mathrm{H}$ plus $\mathrm{He}$ ). Our estimate agrees with this value within our error limits. The relatively minor $\mathrm{He}$ enhancement is consistent with characteristic of Ofc spectral class (Walborn \& Howarth 2000). 
Table 1. Atmospheric parameters of Cyg OB2 \#11, derived in this work - (1) and in the works of Herrero et al. (2002) - (2) and Mokiem et al. (2005) - (3).

\begin{tabular}{|c|c|c|c|c|c|c|c|c|}
\hline & $\begin{array}{c}T_{\text {eff }}, \\
\mathrm{kK}\end{array}$ & $\begin{array}{c}R_{2 / 3}, \\
\mathrm{R}_{\odot}\end{array}$ & $\begin{array}{c}\log L_{*}, \\
L_{\odot}\end{array}$ & $\begin{array}{c}\dot{M}_{\mathrm{uncl}}, \\
10^{-6} \mathrm{M}_{\odot} \mathrm{yr}^{-1}\end{array}$ & $\begin{array}{c}\dot{M}_{\mathrm{cl}} \\
10^{-6} \mathrm{M}_{\odot} \mathrm{yr}^{-1}\end{array}$ & $f_{\infty}$ & $\begin{array}{c}V_{\infty} \\
\mathrm{km} \mathrm{s}^{-1}\end{array}$ & $\beta$ \\
\hline 1 & $36_{-10}^{+0.5}$ & $20.7_{-14}^{+2}$ & $5.81 \pm 0.035$ & $6 \pm 0.7$ & $1.7 \pm 0.2$ & $0.8_{-0.1}^{+0.2}$ & 2200 & $1.3 \pm 0.1$ \\
\hline 2 & $37 \pm 1.5$ & $22.2^{-1.4}$ & $5.89 \pm 0.05$ & 9.88 & & & 2300 & 0.9 \\
\hline 3 & $36.5_{-0.6}^{+0.4}$ & 22.1 & $5.92 \pm 0.11$ & 7.36 & & & 2300 & 1.03 \\
\hline
\end{tabular}
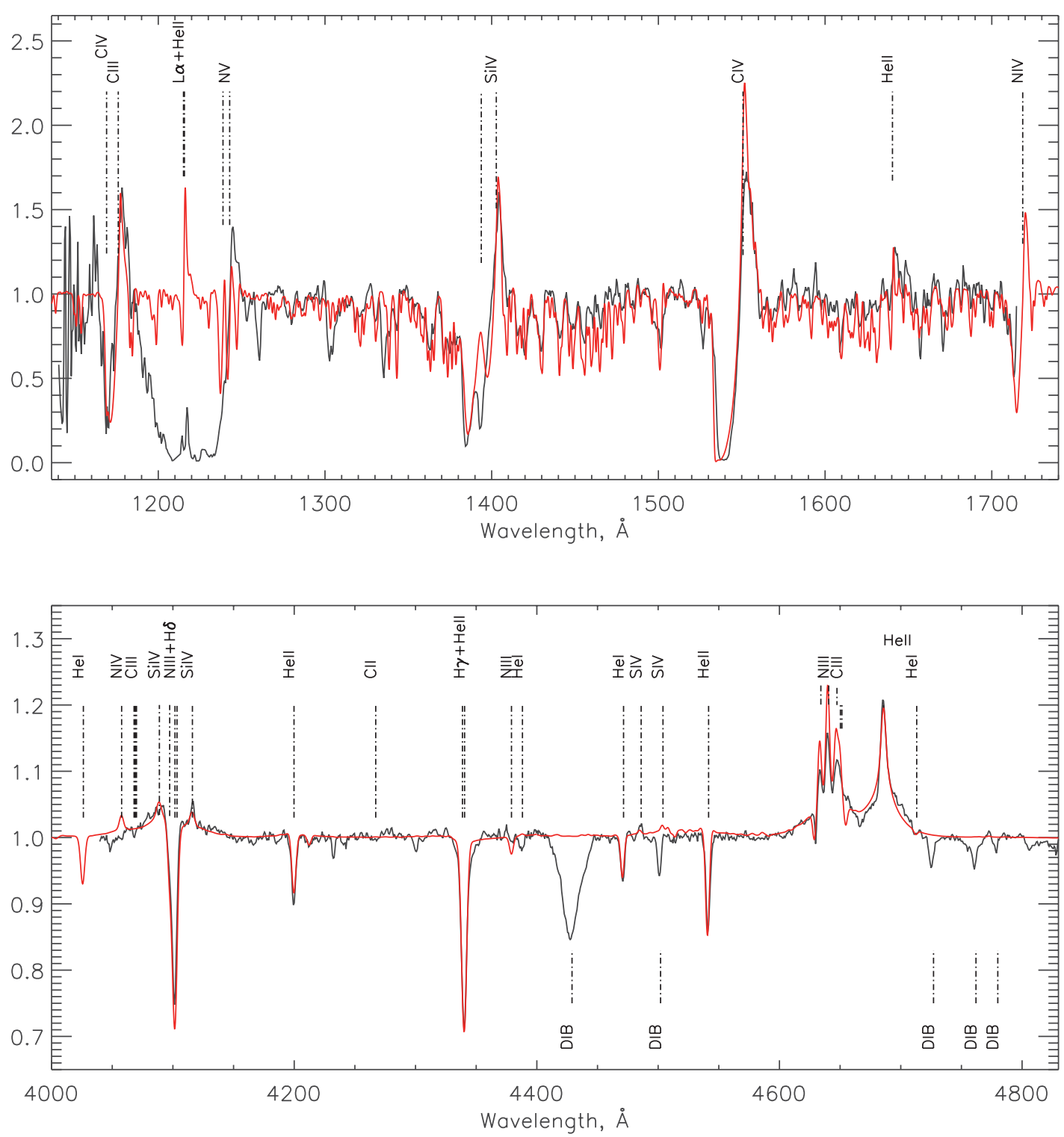

Figure 1. Comparison of the observed spectrum (the black line) with the model (the red line). The top panel shows data obtained by the Hubble Space Telescope, while the bottom panel shows data obtained by the 4.2-m William Herschel Telescope.

Determination of carbon fraction in atmospheres of O-stars is a very important and difficult task. The surface chemical composition depends on the star's rotation rate, metallicity, and mass. Surface chemical abundances are key to understand the physical processes controlling the evolution of massive stars. In optical range there are CIV $\lambda \lambda 5801.3,5812 \AA$, CIII $\lambda \lambda 4647,4650,4652 \AA$, and CIII $\lambda 5696 \AA$ lines. But Martins $\&$ Hillier (2012) do not recommend to use the lines of CIII $\lambda \lambda 4647,4650,4652 \AA$ and CIII $\lambda 5696 \AA$ to determine the carbon abundance, because they are sensitive to $\log g$, effective temperature, mass-loss rate, as well as to the inclusion of other ions in calculations, for example FeIV, FeV, SIV 

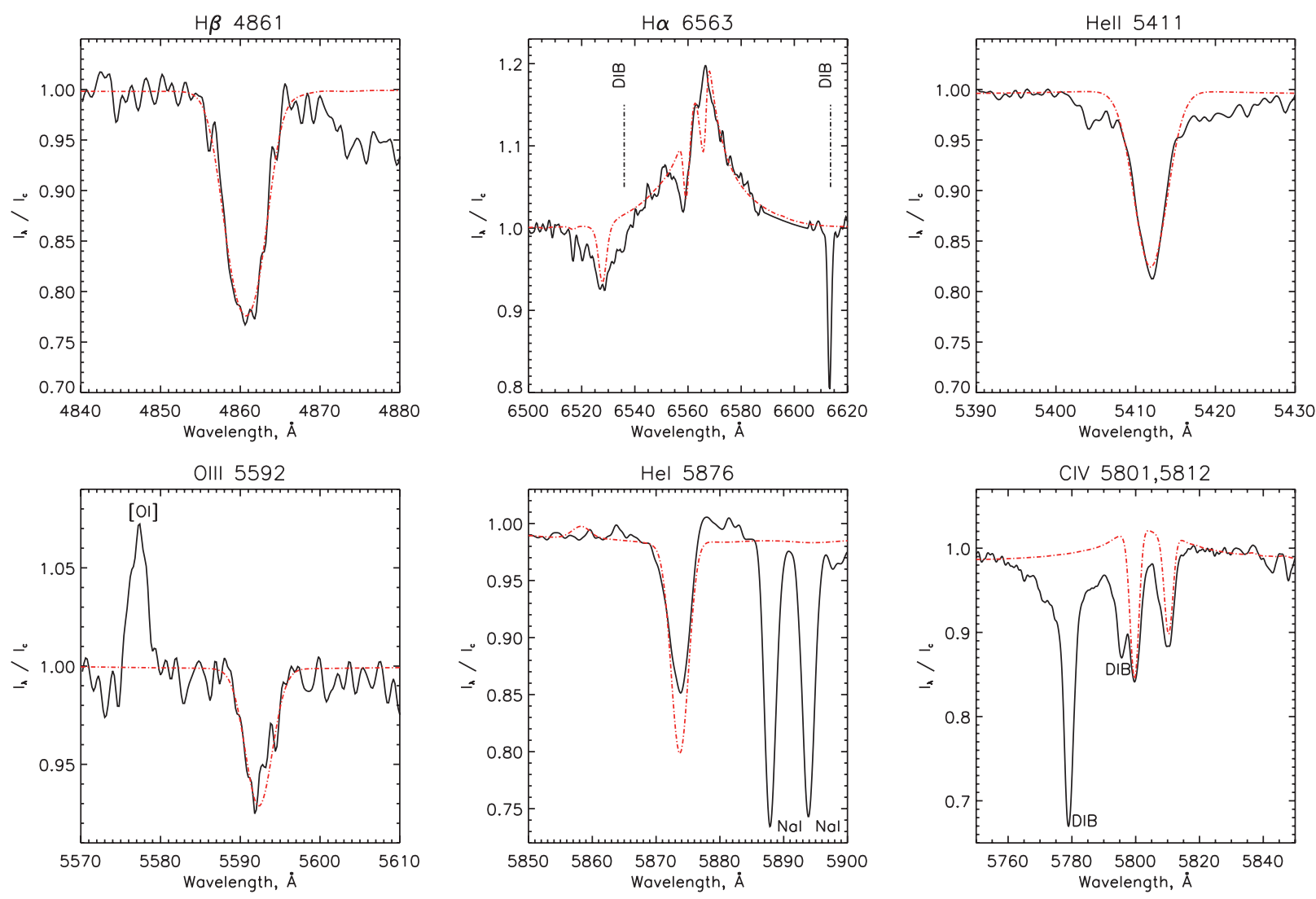

Figure 2. Comparison of the profiles of selected lines with the best model spectra. The black line shows the observed profile, and the red line, the model.

Table 2. The abundances of chemical elements are given in the logarithmic scale relative hydrogen $-\log \left(\mathrm{N}_{\mathrm{el}} / \mathrm{N}_{\mathrm{H}}\right)+12$, where $N_{\mathrm{el}}$ is the abundance of a given element by number.

\begin{tabular}{lrr}
\hline \hline Element & Cyg OB2 \#11 & \multicolumn{1}{c}{ Sun } \\
\hline $\mathrm{H}$ & 12 & 12 \\
$\mathrm{He}$ & $10.85 \pm 0.15$ & $10.93 \pm 0.01$ \\
$\mathrm{C}$ & $8.5 \pm 0.09$ & $8.39 \pm 0.05$ \\
$\mathrm{~N}$ & $8.28 \pm 0.03$ & $7.78 \pm 0.06$ \\
$\mathrm{O}$ & $8.17 \pm 0.07$ & $8.66 \pm 0.05$ \\
$\mathrm{Fe}$ & $7.14 \pm 0.09$ & $7.45 \pm 0.05$ \\
$\mathrm{Si}$ & $7.81 \pm 0.07$ & $7.51 \pm 0.04$ \\
$\mathrm{~S}$ & $6.54 \pm 0.06$ & $7.14 \pm 0.05$ \\
\hline \hline
\end{tabular}

The solar abundances are taken from Grevesse et al. (2005).

(Martins \& Hillier 2012). To determine the carbon abundance in the atmosphere the ultraviolet line CIII $\lambda 1247 \AA$ also may be used (Martins \& Hillier 2012). But, as can be seen in Figure 1, C III 1247 is masked by strong N V emission so it cannot be used for abundance analysis. We determined the carbon abundance in a spectrum of Cyg OB2 \#11 using lines CIV $\lambda \lambda 5801.3,5812 \AA$ and CIII $\lambda 1175 \AA$ (Figures 1 and 2) and we got $\epsilon(\mathrm{C})=12+\log \left[\mathrm{N}_{\mathrm{C}} / \mathrm{N}_{\mathrm{H}}\right]=8.5 \pm 0.09$. Solar abundance of $\mathrm{C}$ is $8.39 \pm 0.05$ (Grevesse, Asplund, \& Sauval 2005), so within the errors, the carbon abundance is solar.
We estimated the nitrogen abundance primarily by emission lines of NIII $\lambda \lambda 4634.0,4640.6 \AA$ and weak absorption of NIV $\lambda \lambda 5200.60,5204.28 \AA$. These lines are considered ideal for determining the nitrogen abundance (Bouret et al. 2012). OIII $\lambda 5592.25 \AA$ line is well distinguishable in the spectrum of the object. It has been used to estimate the oxygen abundance.

In addition to lines of five basis elements $\mathrm{H}, \mathrm{He}, \mathrm{C}, \mathrm{N}$, and $\mathrm{O}$, lines of silicon and sulfur are present in the spectrum of Cyg OB2 \#11. We determined the abundance of silicon using weak absorption SiIV $\lambda \lambda 4629.85,4655.49 \AA$ and emission SiIV $\lambda \lambda 6667.57,6701.21 \AA$ lines. But we were not able to reproduce the doublet of SiIV $\lambda \lambda 1393.75,1402.77 \AA$ lines merging into the strong line with P Cyg profile. This doublet increases with decreasing temperature, but we cannot further decrease the temperature, because emission lines of $\mathrm{He} \mathrm{I}$ lines appear then in optical range, for example, He I $\lambda 4921.94 \AA$, which is not in the spectrum of the object. Silicon doublet SiIV $\lambda \lambda 1393.75,1402.77 \AA$ is also sensitive to the silicon abundance. But to get it, the silicon abundance in atmosphere of Cyg OB2 \#11 should be seven times greater than solar one, which would significantly boost the SiIV optical lines that are also not observed in object spectrum.

We did not use $\mathrm{FeV}$ and FeVI lines to determine the iron abundance in the atmosphere of Cyg OB2 \#11 because there are too many $\mathrm{Fe}$ lines in ultraviolet region and these lines are 
Table 3. Comparison of the atmospheric parameters of Cyg OB2 \#11 with parameters of other O-stars.

\begin{tabular}{lclccccccc}
\hline \hline & Sp. type & $\begin{array}{c}T_{\text {eff }}, \\
\mathrm{kK}\end{array}$ & $\begin{array}{c}L_{*}, \\
10^{5} \mathrm{~L}_{\odot}\end{array}$ & $\begin{array}{c}\dot{M}_{\mathrm{cl}}, \\
\mathrm{M}_{\odot} \mathrm{yr}^{-1}\end{array}$ & $f_{\infty}$ & $\begin{array}{c}V_{\infty}, \\
\mathrm{km} \mathrm{s}^{-1}\end{array}$ & $\begin{array}{c}V \sin I \\
\mathrm{~km} \mathrm{~s}^{-1}\end{array}$ & $\beta$ & Ref. \\
\hline Cyg OB2 \#11 & O5 Ifc & 36 & 6.5 & $1.7 \mathrm{e}-6$ & 0.08 & 2200 & 120 & 1.3 & \\
Cyg OB2 \#8C & O4.5 III(fc) & 37.4 & 3.63 & $2.0 \mathrm{e}-6$ & 0.1 & 2800 & 175 & 1.3 & $\mathrm{a}$ \\
Cyg OB2 \#8A & O5 III(fc) & 37.6 & 13.2 & $3.4 \mathrm{e}-6$ & 0.01 & 2700 & 110 & 1.1 & $\mathrm{a}$ \\
210839 & O6.5 If & 36 & 6.3 & $1.41 \mathrm{e}-6$ & 0.05 & 2100 & 210 & 1. & $\mathrm{~b}$ \\
163758 & O6 I(n)fp & 34.5 & 5.75 & $1.59 \mathrm{e}-6$ & 0.05 & 2100 & 94 & 1.1 & $\mathrm{~b}$ \\
15570 & O4 If & 38 & 8.7 & $2.19 \mathrm{e}-6$ & 0.05 & 2200 & 97 & 1.1 & $\mathrm{~b}$ \\
14947 & O4.5 If & 37 & 6.76 & $1.41 \mathrm{e}-6$ & 0.03 & 2300 & 130 & 1.3 & $\mathrm{~b}$ \\
Obj14 & O5/6 If & 32.5 & 5.8 & $0.37 \mathrm{e}-6$ & & 300 & & 1.0 & $\mathrm{c}$ \\
Obj3 & O5/6 If & 30 & 3.7 & $2.71 \mathrm{e}-6$ & & 800 & & 0.8 & $\mathrm{c}$ \\
\hline \hline
\end{tabular}

${ }^{*}$ Spectral classes are listed according to Sota et al. (2011) catalogue.

a -Najarro, Hanson, \& Puls (2011), b - Bouret et al. (2012), c - Borissova et al. (2012).

$V \sin I$ for Cyg OB2 \#11 are taken from Herrero et al. (2001).
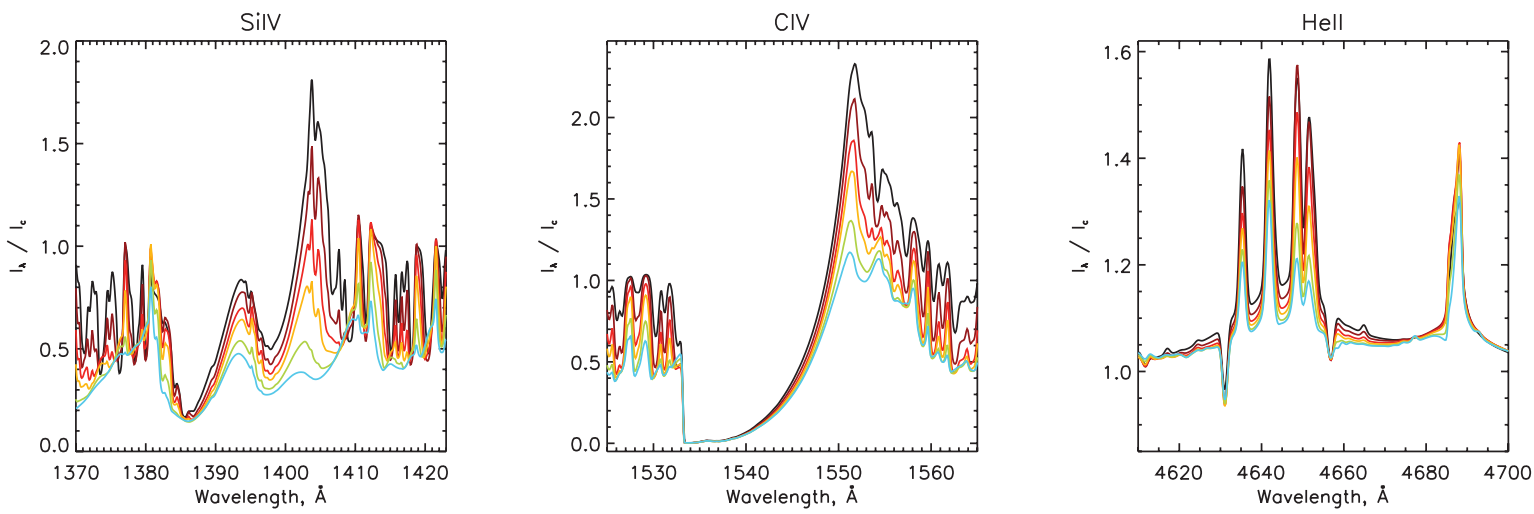

Figure 3. The variation of the Si IV $\lambda \lambda 1393.75,1402.77 \AA$, C IV $\lambda \lambda 1548.2,1550.8 \AA$, and He II 4685.7 line profile depending on the iron abundance. Black line shows the model with $\mathrm{Fe}_{*} / \mathrm{Fe}_{\odot}=0.37$, cherry $-\mathrm{Fe}_{*} / \mathrm{Fe}_{\odot}=1.5$, red $-\mathrm{Fe}_{*} / \mathrm{Fe}_{\odot}=3.7$, orange $-\mathrm{Fe}_{*} / \mathrm{Fe}_{\odot}=6.7$, green $-\mathrm{Fe}_{*} / \mathrm{Fe}_{\odot}=14.8$, and blue $-\mathrm{Fe}_{*} / \mathrm{Fe}_{\odot}=24.3$.

blended into wide bands at low spectral resolution. We used an indirect method instead. The resonance doublet of SiIV $\lambda \lambda 1393.75,1402.77 \AA$ is sensitive to many factors, including Fe abundance. It becomes weaker when iron content in the atmosphere increases (see Figure 3). We found that the SiIV $\lambda \lambda 1393.75,1402.77 \AA$ lines in the spectrum of Cyg OB2 \#11 may be described if we significantly reduce (down to $0.37 \mathrm{X}_{\mathrm{Fe}_{\odot}}$ ) the abundance of iron in the object atmosphere.

\section{LOCATIONS OF Cyg OB2 \#11 ON THE HERTZSPRUNG-RUSSELL DIAGRAM}

In Table 3 the parameters of Cyg OB2 \#11 are compared with the parameters of other O-stars of similar spectral classes. Figure 4 shows the locations of these stars on the Hertzsprung-Russell diagram as well as evolutionary tracks and isochrones from the Geneva database (Ekström et al. 2012), constructed using the online calculator ${ }^{4}$. The evolutionary tracks and isochrones are computed taking into account the effects of rotation. The rotation rate is $v_{\text {ini }} / v_{\text {crit }}=0.4$. It can be seen that Cyg OB2 \#11 is closest

\footnotetext{
${ }^{4}$ http://obswww.unige.ch/Recherche/evol/-Database-
}

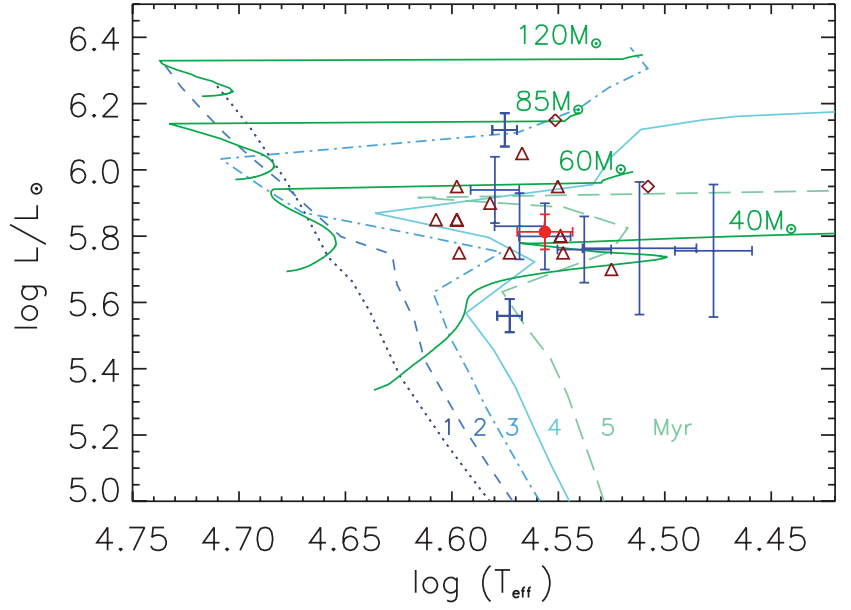

Figure 4. The location of Cyg OB2 \#11 in the Hertzsprung-Russell diagram (marked with the red circle with error bars). For comparison the stars of similar spectral class are shown. Blue points with error bars show stars listed in Table 3. Triangles are 'normal' O4-6 supergiants belonging to Arches cluster, diamonds are 'extreme' O4-6 If $^{+}$belonging to Arches cluster. These data were taken from Martins et al. (2008). The solid horizontal lines represent the mass tracks for stellar masses $120,85,60$, and $40 \mathrm{M}_{\odot}$. The vertical solid lines represent stellar isochrones. The evolution tracks and the stellar isochrones are taken from the Geneva library. 


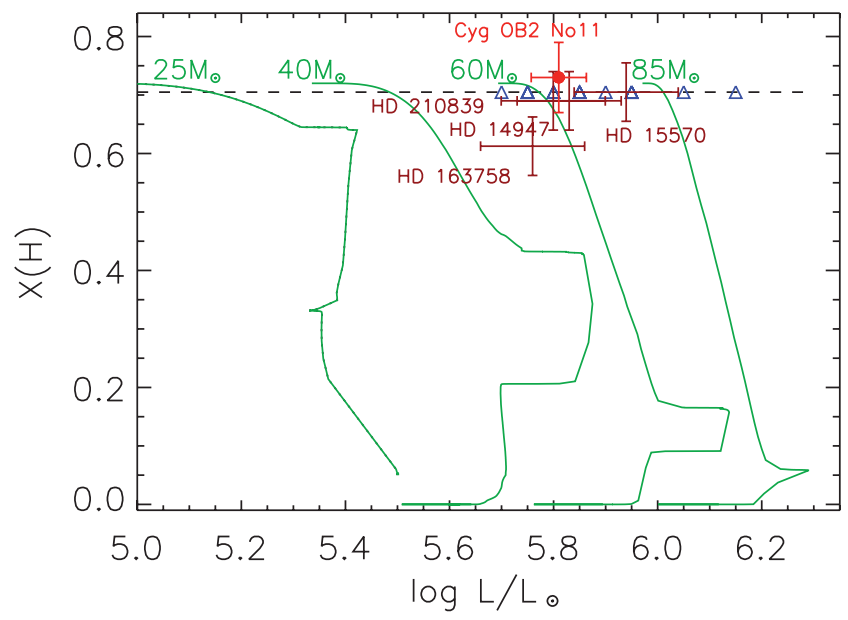

Figure 5. Hydrogen mass-fraction as a function of luminosity. Cyg OB2 \#11 are marked with the red circle with error bars. Locations of O4-6 and $\mathrm{O} 4-6 \mathrm{If}^{+}$supergiants belonging to Arches cluster are marked by triangles. Solid lines show evolution tracks from Geneva library.

to star HD163758 (O6 I(n)fp) and HD210839 (O6.5 If) and it is sufficiently far from two other $\mathrm{O}(\mathrm{fc})$ stars Cyg OB2 \#8 $\mathrm{C}$ and Cyg OB2 \#8 A that are also marked on the diagram. The figure shows that the object lies between the isochrones corresponding to 4 and $5 \mathrm{Myr}$ and the mass of the object is $40-60 \mathrm{M}_{\odot}$. Chentsov et al. (2013) spectroscopically confirmed age differences for stars which are located in different parts of Cyg OB2. Stars located at north part of the association are older than all other objcts in the Cyg OB2. Their ages are about $5 \mathrm{Myr}$ (Chentsov et al. 2013). The results of our numerical simulations support this conclusion and are consistent with the hypothesis of cascade star formation in the association Cyg OB2 (Maryeva, Klochkova, \& Chentsov 2013b).

Figure 5 shows the change of hydrogen mass fraction with age. According to this diagram the mass of Cyg OB2 \#11 is $60 \mathrm{M}_{\odot}$. The figure shows that the object, like HD15570, is on the early part of its evolution track unlike stars HD163758 (O6.5 If) and HD210839 (O6 I(n)fp). It should be noted that although strong CIII lines are observed in the spectra of HD15570 and HD14947, they are weaker than the NIII lines, and these stars are not Ofc stars (Walborn et al. 2010).

Consider the location of Cyg OB2 \#11 on the diagram of carbon mass-fraction as function of nitrogen mass-fraction (Figure 6). This figure shows the evolutionary tracks for stars with masses of $40 \mathrm{M}_{\odot}, 60 \mathrm{M}_{\odot}$, and $85 \mathrm{M}_{\odot}$, computed in 2013 (Ekström et al. 2012). Maeder et al. (2009) write that $N / C$ ratio is sensitive to many parameters such as the age of the star, metallicity, rotation velocity, magnetic field. Evolutionary tracks computed in 2013 are below the tracks designed in 2003 for the same stellar masses (Meynet \& Maeder 2003). Cyg OB2 \#11 is located above the evolutionary tracks. While considering the carbon abundance there are some objects on this diagram similar to Cyg OB2 \#11, but as Figure 6 shows, the nitrogen abundance in this object is



Figure 6. Carbon mass-fraction as function of nitrogen mass-fraction, as predicted by Geneva model with the rotation rate $V_{\text {rot }} / V_{\text {crit }}=0.4$. Solid line is for $40 \mathrm{M}_{\odot}$, dashed for $60 \mathrm{M}_{\odot}$, dotted for $85 \mathrm{M}_{\odot}$. Unnamed triangles mark positions of O4-6 and O4-6 If $^{+}$supergiants belonging to Arches cluster.

lower than that of other O4-6 supergiants. The abundance $\epsilon(N)=8.28 \pm 0.03$ obtained by us is lower than one for 'normal' O4-6 supergiants. Also, it is lower than the nitrogen abundance in other supergiant belonging to Cyg OB2, Cyg OB2 \#7 $\left(\mathrm{O}^{2} \mathrm{II}_{*}, \epsilon(N)=8.65 \pm 0.05\right.$; Maryeva et al. 2013a). Walborn et al. (2000) suggested the hypothesis that the morphologically normal majority of $\mathrm{OB}$ supergiants may be nitrogen enhanced, while the OBC minority have normal $\mathrm{CNO}$ abundances and the OBN class displays more extreme degrees of processed material mixed into the atmospheres. In general, our result is consistent with the hypothesis. Based on the calculations we conclude that nitrogen abundance in OBC stars can be up to two times greater than solar.

\section{SEARCH FOR THE BINARY COMPANION OF Cyg OB2 \#11}

As mentioned above, Cyg OB2 \#11 is a SB1 binary star (Kobulnicky et al. 2012), with period of $72.43 \pm 0.07 \mathrm{~d}$ and $e=0.5 \pm 0.06$. It is known that the fraction of binary and multiple stars among young early-type objects is about $80 \%$ or more, that is more than twice above that for old low-mass stars. Moreover, most probable multiplicity of a newly forming stellar system is $2-3$ (Goodwin \& Kroupa 2005). Thus, even among the field stars the fraction of the triples and systems of higher multiplicity is approximately 0.25 of the total number of binaries. All this makes it reasonable to search for the companions of objects like Cyg OB2 \#11. The search for companions of Cyg OB2 \#11 was already performed by Maíz Apellániz (2010). Maíz Apellániz (2010) has not found companions in the range of separations of $0.1-14$ arcsec and the magnitude differences lower than 8 mag.

So, we performed observations on 6-m Russian BTA telescope with the speckle interferometer, mounted in the primary focus. This device allows to raise the angular 
resolution up to the diffraction limit which is 0.02 arcsec for BTA.

Observations with speckle interferometer based on electron multiplying charge-coupled device (EMCCD; Maksimov et al. 2009) were carried out in the visual spectral range with filters having central wavelengths of 5500 and $8000 \AA$ and pass-band halfwidths of 200 and $1000 \AA$, respectively. We estimated the atmospheric seeing from the full width at half maximum averaged over a sequence of speckle frames for a stellar image, and found it to be about 2 arcsec.

2000 frames of speckle images were accumulated in each series, with single frame exposure of $20 \mathrm{~ms}$. Methods of reduction of speckle interferometric data from BTA is described in the articles Maksimov et al. (2009); Balega et al. (2002). Method of the companion detection is based on analysis of the power spectrum averaged over sequence of speckle interferograms. We report the non-detection of components within separation range of $0.02-4$ arcsec and with magnitude difference less than $6 \mathrm{mag}$ at given wavelengths. Indeed, Cyg OB2 \#11 is extremely bright, so, the chance to find a companion that is 250 times weaker is little (but not zero!). Let us mention the fact that at Cyg OB2 \#11's distance (1.51 kpc according to Kiminki et al. (2007)), 0.02 arcsec separation corresponds to $30 \mathrm{AU}$. So, there is no wide component in this system. And to fill the gap between the direct components detection at the largest telescopes diffraction limit and ability to detect $\mathrm{SB}$, we need to improve angular resolution down to at least $0.001 \mathrm{arcsec}$. The search may be continued in IR range when the instrumental opportunities of high $\Delta$ mag detection will be improved.

\section{RESULTS}

In this work we investigated the atmosphere of O5 Ifc supergiant Cyg OB2 \#11 using the medium-resolution spectra, obtained at RTT15O and WHT, and archival UV data. We have applied non-LTE CMFGEN code for modelling of the atmosphere and determined its physical parameters and chemical composition. Parameters of Cyg OB2 \#11 are similar to the ones of other O4-6 supergiants. The position on the Hertzsprung-Russell diagram corresponds to the mass of star about $50 \mathrm{M}_{\odot}$ and age about 4.5 Myr. Our estimation of age is consistent with ages of other stars located at north part of Cyg OB2.

We found that in the atmosphere of stars $\mathrm{He} / H \approx 0.1$ and nitrogen abundance is lower than that for other "normal' O stars $(\epsilon(N)=8.28 \pm 0.03)$, while the carbon abundance is solar. Overall, our modelling confirms the hypothesis suggested by Walborn (1976); Walborn et al. (2000), that $\mathrm{OBC}$ supergiants have normal $\mathrm{CNO}$ abundances and anomalies in $\mathrm{N}$ versus $\mathrm{C}$, $\mathrm{O}$ correlate with the $\mathrm{He}$ abundance (Walborn \& Howarth 2000). In the spectrum of Cyg OB2 \#11 there are lines of silicon and sulphur, which were used for estimating the abundances of these elements, as results $\epsilon(S i)=7.81 \pm 0.07$ and $\epsilon(S)=6.54 \pm 0.06$.
The speckle interferometry performed on the Russian 6-m BTA telescope does not reveal any binary companion in the range of separations of $0.02-4$ arcsec and with magnitude differences less than $6 \mathrm{mag}$.

\section{ACKNOWLEDGEMENTS}

We would like to thank John D. Hillier for his great code CMFGEN. Likewise, we thank Nolan Walborn for valuable discussions and for providing the data obtained at WHT. We would like to thank the anonymous referee for valuable comments. We used the data from the Multimission Archive at STScl (MAST), the CASU Astronomical Data Centre, SIMBAD database, and database of stellar evolution group at the Geneva Observatory. The study was supported by the Russian Foundation for Basic Research (project nos. 1402-31247, 12-07-00739, 12-02-00185-a, 13-02-00419-a, and 1202-97006-r-povolzhye-a). The observations at the 6-m telescope are supported by the Ministry of Education and Science of Russian Federation (state contract no. 14.518.11.7070). Olga Maryeva thanks the grant of Dynasty Foundation.

\section{REFERENCES}

Balega, I. I., Balega, Y. Y., Hofmann, K.-H., Maksimov, A. F., Pluzhnik, E. A., Schertl, D., Shkhagosheva, Z. U., \& Weigelt, G., 2002, A\&A, 385, 87

Borissova, J., et al. 2012, A\&A, 546, A110

Bouret, J.-C., Hillier, D. J., Lanz, T., \& Fullerton, A. W., 2012, A\&A, 544, A67

Chentsov, E. L., Klochkova, V. G., Panchuk, V. E., Yushkin, M. V., Nasonov, D. S. 2013, Astron. Rep. 57, 527

Ekström, S., et al. 2012, A\&A, 537, A146

Galazutdinov, G. A. 1996, DECH 2.0, Preptint of the Special Astrophysical Observatory

Goodwin, S. P., \& Kroupa, P. 2005, A\&A, 439, 565

Grevesse, N., Asplund, M., \& Sauval, A., 2005, SSRv, 130, 105

Herrero, A., Corral, L. J., Villamariz, M. R., \& Martin, E. L., 1999, A\&A, 348, 542

Herrero, A., Puls, J., Corral, L. J., Kudritzki, R. P., \& Villamariz, M. R., 2001, A\&A, 366, 623

Herrero, A., Puls, J., \& Najarro, F., 2002, A\&A, 396, 949

Hillier, D. J., \& Miller, D. L., 1998, ApJ, 496, 407

Johnson, H. L., \& Morgan, W. W., 1954, ApJ, 119, 344

Kiminki, D. C., et al. 2007, ApJ, 664, 1102

Kobulnicky, H. A., et al. 2012, ApJ, 756, 50

Maeder, A., \& Meynet, G. 2010, NewAR, 54, 32

Maeder, A., Meynet, G., Ekstrom, S., \& Georgy, C., 2009, CoAst, 158,72

Maíz Apellániz, J., 2010, A\&A, 518, A1

Martins, F., \& Hillier, D. J., 2012, A\&A, 545, 95

Martins, F., Hillier, D. J., Paumard, T., Eisenhauer, F., Ott, T., \& Genzel, R., 2008, A\&A, 478, 219

Martins, F., Schaerer, D., \& Hillier, D. J., 2005, A\&A, 436, 1049

Maryeva, O. V., Klochkova, V. G., \& Chentsov, E. L., 2013a, AstBu, 68,87

Maryeva, O. V., Klochkova, V. G., \& Chentsov, E. L. 2013b, arXiv: 1309.4495

Maryeva, O. V., \& Zhuchkov, R. Y., 2012, Ap, 55, 371 
Massey, P., \& Thompson, A. B., 1991, AJ, 101, 1408

Maximov, A. F., Balega, Y. Y., Dyachenko, V. V., Malogolovets, E. V., Rastegaev, D. A., \& Semernikov, E. A. 2009, AstBu, 64, 308

Mel'Nik, A. M., \& Dambis, A. K., 2009, MNRAS, 400, 518

Meynet, G., \& Maeder, A., 2003, A\&A, 404, 975

Mokiem, M. R., de Koter, A., Puls, J., Herrero, A., Najarro, F., \& Villamariz, M. R., 2005, A\&A, 441, 711

Münch, L., \& Morgan, W. W., 1953, AJ, 118, 162

Najarro, F., Hanson, M. M., \& Puls, J., 2011, A\&A, 535, A32

Puls, J., Markova, N., Scuderi, S., Stanghellini, C., Taranova, O. G., Burnley, A. W., \& Howarth, I. D. 2006, A\&A, 454, 625

Puls, J., Urbaneja, M. A., Venero, R., Repolust, T., Springmann, U., Jokuthy, A., \& Mokiem, M. R., 2005, A\&A, 435, 669

Santolaya-Rey, A. E., Puls, J., \& Herrero, A., 1997, A\&A, 323, 488
Sota, A., Maíz Apellániz, J., Walborn, N. R., Alfaro, E. J., Barbá, R. H., Morrell, N. I., Gamen, R. C., \& Arias, J. I., 2011, ApJS, 193, 24

Walborn, N. R., 1973, ApJ, 180, L35

Walborn, N. R., 1976, ApJ, 205, 419

Walborn, N. R., 2003, ASPC, 304, 29

Walborn, N. R., \& Howarth, I. D., 2000, PASP, 112, 1446

Walborn, N. R., Lennon, D. J., Heap, S. R., Lindler, D. J., Smith, L. J., Evans, C. J., \& Parker, J. WM. 2000, PASP, 112, 1243

Walborn, N. R., Morrell, N.I., Howarth, I. D., Crowther, P. A., Lennon, D. J., Massey, P., Arias, J. I., 2004, ApJ, 608, 1028

Walborn, N. R., Sota, A., Maíz Apellániz, J., Alfaro, E. J., Morrell, N. I., Barba, R. H., Arias, J. I., \& Gamen, R. C., 2010, ApJL, 711, L143 